\title{
Passive Radar Imaging of Moving Targets with Sparsely Distributed Receivers
}

\author{
Ling Wang and Birsen Yazıc1** \\ Department of Electrical, Computer and Systems Engineering,Rensselaer Polytechnic Institute, \\ 110 8th Street, Troy, NY 12180 USA \\ Email: yazici@ecse.rpi.edu
}

\begin{abstract}
We develop a novel passive image formation method for moving targets using measurements from a sparse array of receivers that rely on illumination sources of opportunity. We use a physics-based approach to model the wave propagation and develop a passive measurement model that expresses the measurement at each receiver in terms of the measurement at a different receiver. This model eliminates the need for knowledge about the transmitter locations and waveforms in the proposed image formation method. We formulate the image formation problem as a Generalized Likelihood Ratio Test (GLRT) for unknown target location and velocity using the proposed passive measurement model. We form the image in spatial and velocity space using the space- and velocity-resolved test-statistics.
\end{abstract}

\section{INTRODUCTION}

With the rapid growth of broadcasting stations, mobile phone base stations, communication and navigation satellites, as well as relatively low cost and rapid deployment of receivers, passive radar imaging using transmitters of opportunity has emerged as an active area of research in recent years [1]-[7].

In this paper, we develop a passive image formation method for moving targets using static distributed receivers. The method is based on a new passive measurement model and a space- and velocity-resolved binary hypothesis testing. Our method requires no information on the location of transmitters or the transmitted waveforms. Additionally, it does not rely on receivers with high directivity.

\section{Passive Measurement Model}

For a single transmitter with isotropic antenna located at $\mathbf{z}$ transmitting the waveform $p(t)$ starting at time $t=-T_{\mathbf{z}}$, the propagation of electromagnetic waves in a medium can be described using the scalar wave equation,

$$
\left[\nabla^{2}-c^{-2}(t, \mathbf{x}) \partial_{t}^{2}\right] E(t, \mathbf{x}, \mathbf{z})=\delta(\mathbf{x}-\mathbf{z}) p\left(t+T_{\mathbf{z}}\right)
$$

where $c$ is the wave speed in the medium and $E$ is the electric field. Note that this model can be extended to realistic antenna models and multiple transmitters in a straightforward manner.

A single scatterer moving at velocity $\mathbf{v}$ corresponds to an index-of-refraction distribution $n^{2}(\mathbf{x}-\mathbf{v} t)$. Thus, the wave

This work was supported by the Air Force Office of Scientific Research (AFOSR) under the agreements FA9550-07-1-0363 and FA9550-09-1-0013 and National Science Foundation (NSF) under Grant No. CCF-08030672.

** Corresponding author speed $c$ can be expressed as

$$
c^{-2}(t, \mathbf{x})=c_{0}^{-2}\left[1+n^{2}(\mathbf{x}-\mathbf{v} t)\right]
$$

where $c_{0}$ denotes the speed in the background medium and the second term in (2) represents the perturbation due to deviation from the background reflectivity that is caused by the moving scatterer. In this work, we focus specially on radar imaging and assume that $c_{0}$ is equal to the speed of light in vacuum.

Let $q_{\mathbf{v}}(\mathbf{x}-\mathbf{v} t)=c_{0}^{-2} n^{2}(\mathbf{x}-\mathbf{v} t)$. The moving scatterers in the spatial volume $d^{3} x$ centered at $\mathbf{x}$ give rise to $c^{-2}(t, \mathbf{x})=$ $c_{0}^{-2}+\int q_{\mathbf{v}}(\mathbf{x}-\mathbf{v} t) d^{3} v[8]$.

The propagation medium is characterized by the Green's function. For free-space, the Green's function is given by

$$
g(\mathbf{x}, \mathbf{z}, t)=\frac{\delta\left(t-|\mathbf{x}-\mathbf{z}| / c_{0}\right)}{4 \pi|\mathbf{x}-\mathbf{z}|} .
$$

We assume that the electromagnetic waves decay rapidly as they penetrate the ground. We then write $q_{\mathbf{v}}(\mathbf{x}-\mathbf{v} t)$ in terms of $2 \mathrm{D}$ location and $2 \mathrm{D}$ velocity as in

$$
q_{\mathbf{v}}(\mathbf{x}-\mathbf{v} t)=q_{\boldsymbol{v}}(\boldsymbol{x}-\boldsymbol{v} t) \delta\left(x_{3}-h(\boldsymbol{x})\right) \delta\left(v_{3}-D \cdot \boldsymbol{v}\right)
$$

where $\mathbf{x}=\left(\boldsymbol{x}, x_{3}\right), \boldsymbol{x} \in \mathbb{R}^{2}$ and $\mathbf{v}=\left(\boldsymbol{v}, v_{3}\right), \boldsymbol{v} \in \mathbb{R}^{2}, h$ represents the ground topography and $D=\left[\begin{array}{ll}\frac{\partial h}{\partial x_{1}} & \frac{\partial h}{\partial x_{2}}\end{array}\right]$.

For the slow-mover case where the speed of the target is much slower than the speed of light $c_{0}$, the scattered field measurement at the receiver located at $\mathbf{x}_{0}$ due to a transmitter located at $\mathbf{z}$ is expressed as [8]

$$
\begin{aligned}
& m(t)=\int \frac{\ddot{p}\left[\alpha_{\mathbf{y}, \boldsymbol{v}, \mathbf{x}_{0}, \mathbf{z}}\left(t-\left|\mathbf{y}-\mathbf{x}_{0}\right| / c_{0}\right)-|\mathbf{y}-\mathbf{z}| / c_{0}+T_{\mathbf{z}}\right]}{(4 \pi)^{2}\left|\mathbf{y}-\mathbf{x}_{0}\right||\mathbf{y}-\mathbf{z}|} \\
& \times q_{\boldsymbol{v}}(\boldsymbol{y}) d \boldsymbol{y} d \boldsymbol{v}+n(t)
\end{aligned}
$$

where

$$
\alpha_{\boldsymbol{y}, \boldsymbol{v}, \mathbf{x}_{0}, \mathbf{z}}=\frac{1-\widehat{\mathbf{y - z}} \cdot \mathbf{v} / c_{0}}{1+\widehat{\mathbf{y - \mathbf { x } _ { 0 }}} \cdot \mathbf{v} / c_{0}}
$$

is the Doppler-scale-factor [8] and $n(t)$ is the additive thermal noise at the receiver. Note that $\mathbf{y}=(\boldsymbol{y}, h(\boldsymbol{y}))$ and $\mathbf{v}=(\boldsymbol{v}, D$. $\boldsymbol{v})$. In Fourier domain, (5) becomes

$$
\begin{array}{r}
\hat{m}(\omega)=\frac{-\omega^{2}}{(4 \pi)^{2}} \int \frac{\mathrm{e}^{\mathrm{i} \phi_{\boldsymbol{y}, \boldsymbol{v}, \mathbf{x}_{0}}}}{\left|\mathbf{y}-\mathbf{x}_{0}\right||\mathbf{y}-\mathbf{z}| \alpha_{\boldsymbol{y}, \boldsymbol{v}, \mathbf{x}_{0}, \mathbf{z}}^{3}} \hat{p}\left(\frac{\omega}{\alpha_{\boldsymbol{y}, \boldsymbol{v}, \mathbf{x}_{0}, \mathbf{z}}}\right) \\
\times q_{\boldsymbol{v}}(\boldsymbol{y}) d \boldsymbol{y} d \boldsymbol{v}+\hat{n}(\omega) .
\end{array}
$$


where

$$
\phi=\frac{\omega}{\alpha_{\boldsymbol{y}, \boldsymbol{v}, \mathbf{x}_{0}, \mathbf{z}}}\left(T_{\mathbf{z}}-\frac{|\mathbf{y}-\mathbf{z}|}{c_{0}}\right)-\omega \frac{\left|\mathbf{y}-\mathbf{x}_{0}\right|}{c_{0}} .
$$

In the analysis that follows, we consider a sparse distribution of $N$ receivers located at $\mathbf{x}_{i}, i=1, \cdots, N$. We assume that there is a single transmitter located at $\mathbf{z}_{0}$ transmitting waveform $p_{0}$ starting at time $t=-T_{0}$. This assumption allows us to simplify the analysis and distill the important aspects of our imaging theory. The results obtained in this paper can easily be extended to multiple-transmitter case.

Passive measurement model cannot depend on the transmitter location or transmitted waveforms. We develop an alternative measurement model that expresses measurements at each receiver in terms of the measurements at a different receiver, which involves a back-propagation operation and a forward-propagation operation.

Let $u=-q_{\boldsymbol{v}}(\boldsymbol{y}) \omega^{2} \hat{E}^{\mathrm{in}}(\boldsymbol{y}, \boldsymbol{v}, \omega)$ where

$$
\tilde{\hat{E}}^{\text {in }}(\boldsymbol{y}, \boldsymbol{v}, \omega)=\frac{\mathrm{e}^{\mathrm{i} \frac{\omega}{\tilde{\mu} \boldsymbol{y}, \boldsymbol{v}, \mathbf{z}_{0}}\left(T_{0}-\frac{\left|\boldsymbol{y}-\mathbf{z}_{0}\right|}{c_{0}}\right)}}{4 \pi\left|\mathbf{y}-\mathbf{z}_{0}\right| \tilde{\mu}_{\boldsymbol{y}, \boldsymbol{v}, \mathbf{z}_{0}}} \hat{p}_{0}\left(\frac{\omega}{\tilde{\mu}_{\boldsymbol{y}, \boldsymbol{v}, \mathbf{z}_{0}}}\right)
$$

with $\tilde{\mu}_{\boldsymbol{y}, \boldsymbol{v}, \mathbf{z}_{0}}=1-\widehat{\mathbf{y}-\mathbf{z}_{0}} \cdot \mathbf{v} / c_{0}$. Note that $\tilde{\hat{E}}^{\text {in }}$ can be viewed as the incident field observed by a moving target. The scale factor, $\tilde{\mu}$, accounts for the Doppler scaling effect induced by the movement of the target on the field due to the transmitted waveform.

Let $\hat{m}_{i}$ denote the Fourier transform of the measurement at the $i^{\text {th }}$ receiver. Using (7), we define the linear operator, $\mathcal{P}_{\boldsymbol{y}, \boldsymbol{v}, i}$, that propagates the field $u$ to the $i^{\text {th }}$ measurements, i.e.,

$$
\mathcal{P}_{\boldsymbol{y}, \boldsymbol{v}, i}[u](\omega)=\hat{m}_{j}(\omega)
$$

as the forward-propagation operator with respect to the $i^{\text {th }}$ receiver. Using (7), we express $\mathcal{P}_{\boldsymbol{y}, \boldsymbol{v}, i}$ as follows:

$$
\mathcal{P}_{\boldsymbol{y}, \boldsymbol{v}, i}[u](\omega)=\mathcal{G}_{\boldsymbol{y}, i} \mathcal{S}_{\boldsymbol{v}, i}[u](\omega) .
$$

where $\mathcal{S}_{\boldsymbol{v}, i}$ is a scaling operator that accounts for the Dopplerscale factor observed by the $i^{\text {th }}$ receiver due to the movement of the target at the hypothetical velocity $\boldsymbol{v}$; and $\mathcal{G}_{\boldsymbol{y}, i}$ in (11) is a propagation operator that accounts for the time delay from the target to the $i^{\text {th }}$ receiver in free-space.

$\mathcal{S}_{\boldsymbol{v}, i}$ is given by

$$
\mathcal{S}_{\boldsymbol{v}, i}[u](\omega)=\int W_{v}\left(\boldsymbol{v}^{\prime}, \boldsymbol{v}\right) \mu_{\boldsymbol{y}, \boldsymbol{v}^{\prime}, i} u\left(\mu_{\boldsymbol{y}, \boldsymbol{v}^{\prime}, i} \omega\right) d \boldsymbol{v}^{\prime}
$$

where $W_{v}\left(\boldsymbol{v}^{\prime}, \boldsymbol{v}\right)$ is a windowing function of unit amplitude in velocity space centered at the hypothetical velocity $\boldsymbol{v}$ and

$$
\mu_{\boldsymbol{y}, \boldsymbol{v}, i}=1+\widehat{\mathbf{y - \mathbf { x } _ { i }}} \cdot \mathbf{v} / c_{0},
$$

which accounts for the Doppler-scale-factor observed by the $i^{\text {th }}$ receiver due to the movement of the target at the hypothetical velocity $\boldsymbol{v}$.

$\mathcal{G}_{\boldsymbol{y}, i}$ in (11) is given by

$$
\mathcal{G}_{\boldsymbol{y}, i}[u](\omega)=\int W_{s}\left(\boldsymbol{y}^{\prime}, \boldsymbol{y}\right) \hat{g}\left(\mathbf{x}_{i}, \boldsymbol{y}, \omega\right) u\left(\boldsymbol{y}^{\prime}, \boldsymbol{v}, \omega\right) d \boldsymbol{y}^{\prime}
$$

where $W_{s}\left(\boldsymbol{y}^{\prime}, \boldsymbol{y}\right)$ is a spatial windowing function of unit amplitude centered at a hypothetical target location $\boldsymbol{y}$ and $\hat{g}\left(\mathbf{x}_{i}, \boldsymbol{y}, \omega\right)$ represents the Fourier transform of (3).

We define the back-propagation operator as the inverse of $\mathcal{P}_{\boldsymbol{y}, \boldsymbol{v}, i}$ and denote it by $\mathcal{P}_{\boldsymbol{y}, \boldsymbol{v}, i}^{-1}$. Using (11), we obtain

$$
\mathcal{P}_{\boldsymbol{y}, \boldsymbol{v}, i}^{-1}\left[\hat{m}_{i}\right](\omega)=\mathcal{S}_{\boldsymbol{v}, i}^{-1} \mathcal{G}_{\boldsymbol{y}, i}^{-1}\left[\hat{m}_{i}\right](\omega)
$$

where $\mathcal{G}_{\boldsymbol{y}, i}^{-1}$ is the inverse of $\mathcal{G}_{\boldsymbol{y}, i}, \mathcal{S}_{\boldsymbol{v}, i}^{-1}$ is the inverse of $\mathcal{S}_{\boldsymbol{v}, i}$ and $\hat{m}_{i}$ represents the measurement at the $i^{\text {th }}$ receiver. Note that if $\mathcal{P}_{\boldsymbol{y}, \boldsymbol{v}, i}^{-1}$ does not exist, it may be replaced with its pseudoinverse.

We now express the measurement at the $i^{\text {th }}$ receiver, $\hat{m}_{i}$ in terms of the measurement at the $j^{\text {th }}$ receiver, $\hat{m}_{j}$, by back-propagating $\hat{m}_{j}$ measured at $\mathbf{x}_{j}$ to a hypothetical target location with a hypothetical velocity via the back-propagation operator and then forward-propagating the resulting field to $\mathbf{x}_{i}$ via the forward-propagation operator. Let $\hat{m}_{i}^{j}(\omega)$ denote the $i^{\text {th }}$ measurement expressed in terms of the $j^{\text {th }}$ measurement. We define the passive measurement model for the $j^{\text {th }}$ measurement as follows:

$$
\hat{m}_{i}^{j}(\omega)=\mathcal{P}_{\boldsymbol{y}, \boldsymbol{v}, i} \mathcal{P}_{\boldsymbol{y}, \boldsymbol{v}, j}^{-1} \hat{m}_{j}(\omega)+\hat{n}_{i}(\omega)
$$

where $\hat{n}_{i}(\omega)$ is the additive thermal noise at the $i^{\text {th }}$ receiver.

Taking the $j^{\text {th }}$ receiver as a reference, we form the following measurement vector for $N$ receivers:

$$
\mathbf{m}=\left[\begin{array}{llll}
\hat{m}_{1}^{j} & \hat{m}_{2}^{j} & \cdots & \hat{m}_{N}^{j}
\end{array}\right]^{T}
$$

where $\hat{m}_{i}^{j}, i=1, \cdots, N$ and $i \neq j$, denotes the measurement $\hat{m}_{i}$ modeled in terms of the reference measurement $\hat{m}_{j}$. Similarly, we vectorize the "reference measurements", $\hat{m}_{j}$ 's, and the noise:

$$
\begin{aligned}
\mathbf{m}_{\mathbf{r}} & =\left[\begin{array}{llll}
\hat{m}_{j} & \hat{m}_{j} & \cdots & \hat{m}_{j}
\end{array}\right]^{T} \\
\mathbf{n} & =\left[\begin{array}{llll}
\hat{n}_{1} & \hat{n}_{2} & \cdots & \hat{n}_{N}
\end{array}\right]^{T} .
\end{aligned}
$$

Note that $\mathbf{m}, \mathbf{m}_{\mathbf{r}}$ and $\mathbf{n}$ are all $(N-1)$ vectors.

We represent the composition of the back-propagation and forward-propagation operators as a diagonal matrix as follows:

$$
\mathbf{P}_{\boldsymbol{y}, \boldsymbol{v}}=\operatorname{diag}\left[\begin{array}{lll}
\mathcal{P}_{\boldsymbol{y}, \boldsymbol{v}, 1} \mathcal{P}_{\boldsymbol{y}, \boldsymbol{v}, j}^{-1} & \cdots & \mathcal{P}_{\boldsymbol{y}, \boldsymbol{v}, N} \mathcal{P}_{\boldsymbol{y}, \boldsymbol{v}, j}^{-1}
\end{array}\right]
$$

where $i \neq j$ and $\mathbf{P}_{\boldsymbol{y}, \boldsymbol{v}}$ is $(N-1) \times(N-1)$.

Using (16), (17)-(20), we form the vectorized passive measurement model as follows:

$$
\mathbf{m}(\omega)=\mathbf{P}_{\boldsymbol{y}, \boldsymbol{v}} \mathbf{m}_{\mathbf{r}}(\omega)+\mathbf{n}(\omega)
$$

for some range of $\omega$.

Note that in (21) all the operations are understood to be elementwise.

\section{Image Formation in Spatial and Velocity Space}

We formulate the image formation problem as a test of binary hypothesis, which has its roots in the Generalized Likelihood Ratio Test (GLRT) [9]. We extract a space- and velocity-resolved test-statistic as opposed to reconstructing the unknown quantities of interest themselves due to the limited number of measurements available. The image is then formed 
in $(\boldsymbol{y}, \boldsymbol{v})$ domain by the space- and velocity-resolved teststatistic where the location and the velocity can be identified by thresholding the image.

We define the space- and velocity-resolved binary hypothesis test as follows:

$$
\begin{array}{ll}
\mathcal{H}_{0}: & \mathbf{m}=\mathbf{n} \\
\mathcal{H}_{1}: & \mathbf{m}=\mathbf{P}_{\boldsymbol{y}, \boldsymbol{v}} \mathbf{m}_{\mathbf{r}}+\mathbf{n}
\end{array}
$$

where $\mathbf{P}_{\boldsymbol{y}, \boldsymbol{v}}, \mathbf{m}_{r}, \mathbf{m}$ and $\mathbf{n}$ are as defined in (17)-(21). The null hypothesis states that the measurement is due to noise whereas the alternative hypothesis states that the measurement is due to a target located at $\boldsymbol{y}$ moving with velocity $\boldsymbol{v}$.

Using (16), (18), (19) and (22), we obtain

$$
\begin{aligned}
\mathrm{E}\left[\mathbf{m} \mid \mathcal{H}_{0}\right] & =\mathbf{0} \\
\operatorname{Cov}\left[\mathbf{m} \mid \mathcal{H}_{0}\right] & =\mathbf{R}_{\mathbf{n}}=: \mathbf{R}_{0} \\
\mathrm{E}\left[\mathbf{m} \mid \mathcal{H}_{1}\right] & =\mathbf{P}_{\boldsymbol{y}, \boldsymbol{v}} \mathrm{E}\left[\mathbf{m}_{\mathbf{r}} \mid \mathcal{H}_{1}\right]=\mathbf{P}_{\boldsymbol{y}, \boldsymbol{v}} \overline{\mathbf{m}}_{\mathbf{r}} \\
\operatorname{Cov}\left[\mathbf{m} \mid \mathcal{H}_{1}\right] & =\mathbf{P}_{\boldsymbol{y}, \boldsymbol{v}} \mathbf{R}_{\mathbf{n}_{\mathbf{r}}} \mathbf{P}_{\boldsymbol{y}, \boldsymbol{v}}^{H}+\mathbf{R}_{\mathbf{n}}=: \mathbf{R}_{1}
\end{aligned}
$$

where $\mathrm{E}[\cdot]$ denotes the expectation operator and Cov $[\cdot]$ denotes the covariance operator, $\overline{\mathbf{m}}_{\mathbf{r}}$ denotes $\mathrm{E}\left[\mathbf{m}_{\mathbf{r}} \mid \mathcal{H}_{1}\right], \mathbf{P}_{\boldsymbol{y}, \boldsymbol{v}}^{H}$ denotes the Hermitian transpose of $\mathbf{P}_{\boldsymbol{y}, \boldsymbol{v}}, \mathbf{R}_{\mathbf{n}}$ denotes the autocovariance of the vector $\mathbf{n}$, i.e., $\mathbf{R}_{\mathbf{n}}\left(\omega, \omega^{\prime}\right)=\mathrm{E}\left[\mathbf{n}(\omega) \mathbf{n}^{H}\left(\omega^{\prime}\right)\right]$ and $\mathbf{R}_{\mathbf{n}_{\mathbf{r}}}$ is the autocovariance of the noise vector $\mathbf{n}_{\mathbf{r}}=$ $\left[\hat{n}_{j}, \hat{n}_{j}, \cdots, \hat{n}_{j}\right]$, i.e., $\mathbf{R}_{\mathbf{n}_{\mathbf{r}}}\left(\omega, \omega^{\prime}\right)=\mathrm{E}\left[\mathbf{n}_{\mathbf{r}}(\omega) \mathbf{n}_{\mathbf{r}}^{H}\left(\omega^{\prime}\right)\right]$.

We determine the test-statistic by maximizing the signalto-noise ratio (SNR) of the test-statistic while constraining the associated discriminant functional to be linear. The linear discriminant functional involved in our problem has the form

$$
\lambda=\langle\mathbf{m}, \mathbf{w}\rangle:=\int \mathbf{w}^{H} \mathbf{m} d \omega=\sum_{i \neq j} \int w_{i}^{*}(\omega) \hat{m}_{i}^{j}(\omega) d \omega
$$

where $\lambda$ denote the the output of the discriminant functional, which we call the test-statistic and $\mathbf{w}$ is a template given by $\mathbf{w}=\left[\begin{array}{ll}w_{1} & w_{2} \cdots w_{N}\end{array}\right]^{T}$.

Under the assumption that the noise at different receivers are wide sense stationary and mutually uncorrelated, the maximization of the SNR of $\lambda$ [9] results in an optimal linear template given by

$$
\mathbf{w}^{\star}=\overline{\mathbf{S}}^{-1}(\omega) \mathbf{P}_{\boldsymbol{y}, \boldsymbol{v}}(\omega) \overline{\mathbf{m}}_{\mathbf{r}}(\omega)
$$

where $\overline{\mathbf{S}}^{-1}$ is the inverse of $\overline{\mathbf{S}}$ defined by $\overline{\mathbf{S}}(\omega)=$ $\int 1 / 2\left(\mathbf{R}_{1}\left(\omega, \omega^{\prime}\right)+\mathbf{R}_{0}\left(\omega, \omega^{\prime}\right)\right) d \omega^{\prime}$. Using (24) and (26), We can approximate $\overline{\mathbf{S}}^{-1}$ by a diagonal matrix. (See [10] for details.) We denote diagonal elements of $\overline{\mathbf{S}}^{-1}$ by $\bar{S}_{i}^{-1}(\omega), i=$ $1, \cdots, N$ and $i \neq j$, which is a function of the power spectral density function of noise at the $i^{\text {th }}$ receiver and the kernel of $\mathbf{P}_{\boldsymbol{y}, \boldsymbol{v}}$.

\section{Resolution Analysis}

In this section, we assume that the surface topography is flat, i.e., $h(\boldsymbol{y})=h$, for some $\boldsymbol{y} \in \mathbb{R}^{2}$ and therefore set $\mathbf{y}=[\boldsymbol{y}, h]$, $\mathbf{v}=[\boldsymbol{v}, 0]$. We focus our analysis on the deterministic moving point target model given by

$$
q_{\boldsymbol{v}}(\boldsymbol{y})=T \delta\left(\boldsymbol{y}-\boldsymbol{y}_{0}\right) \delta\left(\boldsymbol{v}-\boldsymbol{v}_{0}\right)
$$

and analyze how moving point targets are resolved in the fourdimensional image $\lambda(\boldsymbol{y}, \boldsymbol{v}), \boldsymbol{y}, \boldsymbol{v} \in \mathbb{R}^{2}$.

Let $K\left(\boldsymbol{y}, \boldsymbol{y}_{0} ; \boldsymbol{v}, \boldsymbol{v}_{0}\right)$ denote the PSF of the four-dimensional imaging operator. We define $K$ as the expected value of the image of a moving point target represented by (29), i.e., $K\left(\boldsymbol{y}, \boldsymbol{y}_{0} ; \boldsymbol{v}, \boldsymbol{v}_{0}\right):=\mathrm{E}[\lambda(\boldsymbol{y}, \boldsymbol{v})]$.

Without loss of generality, we first assume that there is a single pair of receivers present in the scene and then extend the results to the case where there are multiple pairs of receivers.

For a deterministic moving point target model given by (29), we have

$$
\begin{aligned}
\mathrm{E}\left[\hat{m}_{j}(\omega)\right]=\frac{\omega^{2} T \mathrm{e}^{\mathrm{i} \phi_{\boldsymbol{y}_{0}, \boldsymbol{v}_{0}, \mathrm{j}}}}{(4 \pi)^{2}\left|\mathbf{y}_{0}-\mathbf{x}_{j}\right|\left|\mathbf{y}_{0}-\mathbf{z}_{0}\right| \alpha_{\boldsymbol{y}_{0}, \boldsymbol{v}_{0}, \mathbf{x}_{j}, \mathbf{z}_{0}}^{3}} \\
\times \hat{p}_{0}\left(\frac{\omega}{\alpha_{\boldsymbol{y}_{0}, \boldsymbol{v}_{0}, \mathbf{x}_{j}, \mathbf{z}_{0}}}\right)
\end{aligned}
$$

where $\alpha_{\boldsymbol{y}_{0}, \boldsymbol{v}_{0}, \mathbf{x}_{j}, \mathbf{z}_{0}}$ is as described by (6) and

$$
\phi_{\boldsymbol{y}_{0}, \boldsymbol{v}_{0}, j}=\frac{\omega}{\alpha_{\boldsymbol{y}_{0}, \boldsymbol{v}_{0}, \mathbf{x}_{j}, \mathbf{z}_{0}}}\left(T_{\mathbf{z}_{1}}-\frac{\left|\mathbf{y}_{0}-\mathbf{z}_{0}\right|}{c_{0}}\right)-\omega \frac{\left|\mathbf{y}_{0}-\mathbf{x}_{j}\right|}{c_{0}} .
$$

For notational simplicity, we drop $\mathbf{z}_{0}$ from the subscript of $\alpha$ for the rest of our paper.

We assume that there are two receivers located at $\mathbf{x}_{1}$ and $\mathbf{x}_{2}$ and take the measurement at the $\mathbf{x}_{1}$ as the reference. Thus, Using(27), (28), (11), (15), (12), (14) and (29), we have

$$
\begin{aligned}
& K\left(\boldsymbol{y}, \boldsymbol{y}_{0} ; \boldsymbol{v}, \boldsymbol{v}_{0}\right)=\frac{\left|\mathbf{y}-\mathbf{x}_{1}\right|}{\left|\mathbf{y}-\mathbf{x}_{2}\right|} \gamma_{\boldsymbol{y}, \boldsymbol{v}, 21} \int \bar{S}_{2}^{-1}(\omega) \times \\
& \mathrm{e}^{-\mathrm{i} k\left(\left|\mathbf{y}-\mathbf{x}_{2}\right|-\gamma_{\boldsymbol{y}, \boldsymbol{v}, 21}\left|\mathbf{y}-\mathbf{x}_{1}\right|\right)} \mathrm{E}\left[\hat{m}_{1}\left(\gamma_{\boldsymbol{y}, \boldsymbol{v}, 21} \omega\right)\right] \mathrm{E}\left[\hat{m}_{2}^{*}(\omega)\right] d \omega
\end{aligned}
$$

where

$$
\gamma_{\boldsymbol{y}, \boldsymbol{v}, 21}=\frac{1+\widehat{\mathbf{y - \mathbf { x } _ { 2 }}} \cdot \mathbf{v} / c_{0}}{1+\widehat{\mathbf{y - x _ { 1 }}} \cdot \mathbf{v} / c_{0}}
$$

Using (30), (32) becomes

$$
\begin{aligned}
K\left(\boldsymbol{y}, \boldsymbol{y}_{0} ; \boldsymbol{v}, \boldsymbol{v}_{0}\right) & =\beta \int \bar{S}_{2}^{-1}(\omega) \omega^{4} \mathrm{e}^{-\mathrm{i} \mathrm{kr}_{21}} \\
\times & \mathrm{e}^{\mathrm{i} k\left[\left(\frac{\gamma_{\boldsymbol{y}, \boldsymbol{v}, 21}}{\alpha \boldsymbol{y}_{0}, \boldsymbol{v}_{0}, \mathbf{x}_{1}}-\frac{1}{\alpha \boldsymbol{y}_{0}, \boldsymbol{v}_{0}, \mathbf{x}_{2}}\right)\left(c T_{\mathbf{z}_{0}}-\left|\mathbf{y}_{0}-\mathbf{z}_{0}\right|\right)\right]} \\
\times & \hat{p}_{0}\left(\frac{\gamma_{\boldsymbol{y}, \boldsymbol{v}, 21}}{\alpha_{\boldsymbol{y}_{0}, \boldsymbol{v}_{0}, \mathbf{x}_{1}}} \omega\right) \hat{p}_{0}^{*}\left(\frac{\omega}{\alpha_{\boldsymbol{y}_{0}, \boldsymbol{v}_{0}, \mathbf{x}_{2}}}\right) d \omega
\end{aligned}
$$

where $\beta$ is a s scaling term due to geometric spreading factors and Doppler-scale-factors and

$r_{21}=\left|\mathbf{y}-\mathbf{x}_{2}\right|-\gamma_{\boldsymbol{y}, \boldsymbol{v}, 21}\left|\mathbf{y}-\mathbf{x}_{1}\right|-\left(\left|\mathbf{y}_{0}-\mathbf{x}_{2}\right|-\gamma_{\boldsymbol{y}, \boldsymbol{v}, 21}\left|\mathbf{y}_{0}-\mathbf{x}_{1}\right|\right)$.

Note that if $T_{\mathbf{z}_{0}}$ is chosen to be equal to $\left|\mathbf{y}_{0}-\mathbf{z}_{0}\right| / c_{0}$, the second exponential term disappears.

Examining (34), we see that if

$$
\frac{\gamma_{\boldsymbol{y}, \boldsymbol{v}, 21}}{\alpha_{\boldsymbol{y}_{0}, \boldsymbol{v}_{0}, \mathbf{x}_{1}}}=\frac{1}{\alpha_{\boldsymbol{y}_{0}, \boldsymbol{v}_{0}, \mathbf{x}_{2}}}
$$


(34) becomes

$$
\begin{aligned}
K\left(\boldsymbol{y}, \boldsymbol{y}_{0}\right. & \left.; \boldsymbol{v}, \boldsymbol{v}_{0}\right)=\frac{T^{2}\left|\mathbf{y}-\mathbf{x}_{1}\right|}{(4 \pi)^{4}\left|\mathbf{y}_{0}-\mathbf{z}_{0}\right|^{2}\left|\mathbf{y}_{0}-\mathbf{x}_{1}\right|\left|\mathbf{y}-\mathbf{x}_{2}\right| \alpha_{\boldsymbol{y}_{0}, \boldsymbol{v}_{0}, \mathbf{x}_{2}}} \\
& \times \int \bar{S}_{2}^{-1}(\omega) \omega^{4} \mathrm{e}^{-\mathrm{i} k r_{21}}\left|\hat{p}_{0}\left(\frac{\omega}{\alpha_{\boldsymbol{y}_{0}, \boldsymbol{v}_{0}, \mathbf{x}_{2}}}\right)\right|^{2} d \omega
\end{aligned}
$$

which defines the correlation of $\left|\bar{S}_{2}^{-1 / 2}(\omega) \omega^{2} \hat{p}_{1}\left(\frac{\omega}{\alpha_{\mathbf{y}_{0}, \boldsymbol{v}_{0}, \mathbf{x}_{2}}}\right)\right|$ with itself in time domain. Clearly, this correlation peaks when $r_{21}=0$, i.e.,

$$
\left|\mathbf{y}-\mathbf{x}_{2}\right|-\gamma_{\boldsymbol{y}, \boldsymbol{v}, 21}\left|\mathbf{y}-\mathbf{x}_{1}\right|=\left|\mathbf{y}_{0}-\mathbf{x}_{2}\right|-\gamma_{\boldsymbol{y}, \boldsymbol{v}, 21}\left|\mathbf{y}_{0}-\mathbf{x}_{1}\right| .
$$

The analysis above shows that the PSF of the imaging operator for two receivers and one transmitter becomes maximum under the two conditions given by (36) and (38).

Substituting (6) into (36), we have

$$
\gamma_{\boldsymbol{y}, \boldsymbol{v}, 21}=\frac{1+\widehat{\mathbf{y}_{0}-\mathbf{x}_{2}} \cdot \mathbf{v}_{0} / c_{0}}{1+\widehat{\mathbf{y}_{0}-\mathbf{x}_{1}} \cdot \mathbf{v}_{0} / c_{0}}=\gamma_{\boldsymbol{y}_{0}, \boldsymbol{v}_{0}, 21} .
$$

Note that $\mathbf{y}_{0}=\left(\boldsymbol{y}_{0}, h\right)$ and $\mathbf{v}_{0}=\left(\boldsymbol{v}_{0}, 0\right)$. Under the slowermover assumption, $\gamma_{\boldsymbol{y}, \boldsymbol{v}, i j}$ can be approximated as follows:

$\gamma_{\boldsymbol{y}, \boldsymbol{v}, i j}=\frac{1+\widehat{\mathbf{y - x _ { i }}} \cdot \mathbf{v} / c_{0}}{1+\widehat{\mathbf{y - x _ { j }}} \cdot \mathbf{v} / c_{0}} \approx 1+\left(\widehat{\mathbf{y - x _ { i }}}-\widehat{\mathbf{y - x _ { j }}}\right) \cdot \mathbf{v} / c_{0}$.

Let

$$
\left\{(\boldsymbol{y}, \boldsymbol{v}) \in \mathbb{R}^{2} \times \mathbb{R}^{2}: \gamma_{\boldsymbol{y}, \boldsymbol{v}, i j}=C\right\}
$$

for some constant $C \in \mathbb{R}^{+}$. Using (40), $\gamma_{\boldsymbol{y}, \boldsymbol{v}, i j}=C$ can be written as

$$
\left(\widehat{\mathbf{y - x _ { 2 }}}-\widehat{\mathbf{y - x _ { 1 }}}\right) \cdot \mathbf{v}=(C-1) c_{0} .
$$

Note that if we multiply both sides by $\omega$, the left-hand side of (42) becomes the hitchhiker-Doppler defined in [11] and for a fixed frequency it becomes the DSAH-Doppler defined in [12]. In this regard, we refer to the four-dimensional manifold formed by $\left\{(\boldsymbol{y}, \boldsymbol{v}) \in \mathbb{R}^{2} \times \mathbb{R}^{2}: \gamma_{\boldsymbol{y}, \boldsymbol{v}, i j}=C\right\}$ in $(\boldsymbol{y}, \boldsymbol{v})$ space, for some constant $C \in \mathbb{R}^{+}$, as a passive-iso-Doppler manifold.

Thus, (39) specifies a passive-iso-Doppler manifold with respect to two receivers located at $\mathbf{x}_{1}$ and $\mathbf{x}_{2}$. The test-statistic due to a moving point target located at $\boldsymbol{y}_{0}$ moving with velocity $\boldsymbol{v}_{0}$ is constant on this mainfold.

Substituting (39) into the left-hand side of (38), we obtain

$$
\left|\mathbf{y}-\mathbf{x}_{2}\right|-\gamma_{\boldsymbol{y}, \boldsymbol{v}, 21}\left|\mathbf{y}-\mathbf{x}_{1}\right|=\left|\mathbf{y}_{0}-\mathbf{x}_{2}\right|-\gamma_{\boldsymbol{y}_{0}, \boldsymbol{v}_{0}, 21}\left|\mathbf{y}_{0}-\mathbf{x}_{1}\right| \text {. }
$$

Let

$$
r_{21}(\boldsymbol{y}, \boldsymbol{v})=\left|\mathbf{y}-\mathbf{x}_{2}\right|-\gamma_{\boldsymbol{y}, \boldsymbol{v}, 21}\left|\mathbf{y}-\mathbf{x}_{1}\right| .
$$

We refer to $r_{21}(\boldsymbol{y}, \boldsymbol{v})$ as passive-range. For a pair of receivers located at $\mathbf{x}_{1}$ and $\mathbf{x}_{2},\left\{(\boldsymbol{y}, \boldsymbol{v}) \in \mathbb{R}^{2} \times \mathbb{R}^{2}: r_{12}(\boldsymbol{v}, \boldsymbol{y})=C\right\}$ where $C \in \mathbb{R}^{+}$denotes some constant forms a manifold in the four-dimensional $(\boldsymbol{y}, \boldsymbol{v})$ space. We refer to the this manifold as an passive-iso-range manifold. (43) specifies an passiveiso-range manifold with respect to two receivers located at $\mathbf{x}_{1}$ and $\mathrm{x}_{2}$. The test-statistic due to a moving point target located at $\boldsymbol{y}_{0}$ moving with velocity $\boldsymbol{v}_{0}$ is constant on this manifold.
Based on the analysis above, we conclude that PSF peaks at the intersection of the passive-iso-Doppler manifold defined by (39), and the passive-iso-range manifold defined by (43). Note that (37) can be interpreted as a generalized autoambiguity function of the transmitted waveform $p_{1}$. The spread of the passive-iso-Doppler manifold and the passive-iso-range manifold are both related to the shape of this generalized autoambiguity function. Hence, the resolution of the reconstructed image in $(\boldsymbol{y}, \boldsymbol{v})$ is determined by the overlapping area between the passive-iso-Doppler and passive-iso-range manifolds.

For $N>2$ receivers, it can be shown that multiple pairs of receivers generate multiple four-dimensional passive-isoDoppler and passive-iso-range manifolds. These manifolds intersect at the correct target location and correct velocity in $(\boldsymbol{y}, \boldsymbol{v})$ space and contribute to the reconstruction of the target image. The test-statistic value at the the correct target location and correct velocity increases by roughly a factor of $N-1$ as compared to the two-receiver scenario as described in (34).

\section{Conclusion}

In this work, we proposed a new passive radar imaging method for moving targets using sparsely distributed receivers. We performed extensive numerical simulations to verify our method. Due to page limitations, numerical simulations will be presented at the conference.

While we mainly focused on passive radar application, the results presented in our paper are also applicable to other wave-based passive imaging applications, such as passive acoustic or seismic imaging.

\section{REFERENCES}

[1] H. D. Griffiths and N. R. W. Long, "Television-based bistatic radar," IEE Proceedings of Radar, Sonar and Navigation, vol. 133, no. 7, pp. 649-657, July 1986.

[2] H. D. Griffiths and C. J. Baker, "Passive coherent location radar systems. part 1: Performance prediction," IEE Proceedings of Radar, Sonar and Navigation, vol. 152, no. 3, pp. 153-159, June 2005.

[3] D. Poullin, "Passive detection using digital broadcasters (dab, dvb) with cofdm modulation," IEE Proceedings of Radar, Sonar and Navigation, vol. 152, no. 3, pp. 143-152, June 2005.

[4] J. Homer, K. Kubik, B. Mojarrabi, I. Longstaff, E. Donskoi, and M. Cherniakov, "Passive bistatic radar sensing with leos based transmitters," Proc. IEEE Int. Geoscience and Remote Sensing Symp., pp. 438-440, 2002.

[5] V. Koch and R. Westphal, "New approach to a multistatic passive radar sensor for air/space defense," IEEE Aero. Electron. Syst. Mag., vol. 10, pp. 24-32, Nov. 1995.

[6] K. S. Kulpa, "Multi-static entirely passive detection of moving targets and its limitations," IEE Proceedings of Radar, Sonar, and Navigation, vol. 152, no. 3, pp. 169-173, June 2005.

[7] H. Guo, K. Woodbridge, and C. J. Baker, "Evaluation of wifi beacon transmissions for wireless based passive radar," in Proc. IEEE Radar Conference, Rome, Italy, 26-30 May 2008.

[8] M. Cheney and B. Borden, "Imaging moving targets from scattered waves," Inverse Problems, vol. 24, pp. 035 005(1-22), 2008.

[9] S. M. Kay, Fundamentals of Statistical Signal Processing, Vol. I and Vol. II. Prentice Hall, 1998.

[10] L. Wang and B. Yazıcı, "Passive imaging of moving targets using distributed apertures," in preparation.

[11] C. E. Yarman and B. Yazıcı, "Synthetic aperture hitchhiker imaging," IEEE Transactions on Imaging Processing, vol. 17, no. 11, pp. 21562173, November 2008.

[12] C. E. Yarman, L. Wang, and B. Yazıcı, "Doppler synthetic aperture hitchhiker imaging," Under review by Inverse Problems, 2009. 